\title{
5G aday frekans bandında 4-Portlu MIMO antenin geliştirilmesi
}

\author{
Development of 4-Port MIMO antenna in the $5 G$ candidate frequency band
}

\author{
Mehmet Ali ÖZASLAN*1,a \\ ${ }^{I}$ Recep Tayyip Erdoğan Üniversitesi, Mühendislik ve Mimarlık Fakültesi, Elektrik ve Elektronik Mühendisliği Bölümü, 53000, Rize
}

• Geliş tarihi / Received: 26.05.2020 • • • Düzeltilerek geliş tarihi / Received in revised form: 31.03.2021 • Kabul tarihi / Accepted: 08.04 .2021

\begin{abstract}
$\ddot{O} z$
$\mathrm{Bu}$ çalışmada, 5G aday frekans bandında 4-portlu masif Çoklu-Giriş-Çoklu-Çıkış (Multiple-Input-Multiple-Output, MIMO) anten tasarımı geliştirilmiştir. MIMO anten yapısı, bir dikdörtgen, bir daire ve bir zemin düzleminden oluşmaktadır. MIMO anten boyutu 35x50x0.76mm3'tür. Antenin bant genişliği S11 parametresine göre $1.1 \mathrm{GHz}$ olarak bulunmuştur. Bant genişliği bu anten için yüksek olup ideal değere sahiptir. MIMO antenin aktif yansıma katsayıları maksimum-14.5dB civarlarında ve zarf korelasyon katsayıları ise 0.017 'den daha küçük bulunmuştur. Antenin $24 \mathrm{GHz}$ 'de maksimum verimlilik değeri $\% 94.50,27 \mathrm{GHz}$ 'de $\% 91.81$ ve $28 \mathrm{GHz}$ 'de $\% 81.81$ olarak elde edilmiştir. Geliştirilen 4-portlu MIMO antenin radyasyon değerleri normalize edilmiş değerlerdir. Antenin tasarımı ve analizi ANSYS HFSS ${ }^{\circledR}$ bilgisayar benzetimi kullanılarak tamamlanmıştır. İncelenen frekans değerleri $5 \mathrm{G}$ teknolojisi için lisanslanmış aday frekans değerleridir. Tasarlanan antenden bulunan kazanç değerleri $24 \mathrm{GHz}$ 'de $12.70 \mathrm{~dB}, 27 \mathrm{GHz}$ 'de $13.05 \mathrm{~dB}$ ve $28 \mathrm{GHz}$ 'de $10.48 \mathrm{~dB}$ olarak bulunmuştur. Bulunan kazanç değerleri 4-portlu MIMO anten için önemli değerlere sahiptir.
\end{abstract}

Anahtar kelimeler: 24GHz, 5G, 6GHz, Anten kazanc1, MIMO i

\begin{abstract}
In this study, 4 port massive Multiple-Input-Multiple-Output (MIMO) antenna design has been developed in the $5 G$ candidate frequency band. The MIMO antenna structure consists of a rectangle, a circle, and a ground plane. MIMO antenna size is $35 \times 50 x 0.76 \mathrm{~mm} 3$. According to the $\$ 11$ parameter, the bandwidth of the antenna was found to be $1.1 \mathrm{GHz}$. The bandwidth is high for this antenna and has an ideal value. The active reflection coefficients of the MIMO antenna were maximum $-14.5 \mathrm{~dB}$ and the envelope correlation coefficient was less than 0.017. The maximum efficiency of the antenna at $24 \mathrm{GHz}$ is $94.50 \%, 91.81 \%$ at $27 \mathrm{GHz}$ and, $81.81 \%$ at $28 \mathrm{GHz}$. The radiation values of the developed 4-port MIMO antenna are normalized values. The design and analysis of the antenna was completed using ANSYS HFSS ${ }^{\circ}$ computer simulator. The frequency values investigated are candidate frequency values licensed for $5 G$ technology. The gain values from the designed antenna are $12.70 \mathrm{~B}$ at $24 \mathrm{GHz}, 13.05 \mathrm{~dB}$ at $27 \mathrm{GHz}$ and, $10.48 \mathrm{~dB}$ at $28 \mathrm{GHz}$. The gain values found have important values for the 4-port MIMO antenna.
\end{abstract}

Keywords: $24 \mathrm{GHz}, 5 \mathrm{G}, 6 \mathrm{GHz}$, Antenna gain, $M I M O$

\footnotetext{
${ }^{{ }^{*} a}$ Mehmet Ali ÖZASLAN; ma.ozaslann@gmail.com, orcid.org/0000-0002-8721-601X
} 


\section{Giriş}

MIMO, öngörülen avantajı sağlamak için çok yollu ortamlar gerektirir. Birden fazla veri akışı göndererek, sistemin güvenilirliğini ve veri hızlarını önemli ölçüde artırmaktadır (Sharawi, 2017). MIMO'da, vericideki çoklu antenlerden birden fazla veri akışı gönderilir ve alıcı ucundaki birden fazla anten tarafından alınır (Rajo-Iglesias ve Sharawi, 2016). 5G teknolojisinin önemli bir parçası olan MIMO, kullanıcılara yüksek veri hızları sunmak için geliştirilmiş bir teknolojidir. Eş zamanlı çoklu iletim için, verici ve alıcı kısımlarında çoklu anten sistemleri gereklidir. Bununla beraber neredeyse $6 \mathrm{GHz}$ alt1 frekans bantları için spektrum dolmuştur. Bundan dolayı 5G'de yüksek frekanslara çıkılması amaçlanmıştır. Yüksek frekans değerlerine çıkılması, dalga boyunun küçüleceği anlamına gelir. Dalga boyu ile anten boyu arasında bir ilişki söz konusudur. Bu, tasarlanan antenin boyutunun küçüleceğini yani milimetre seviyelerine düşeceği anlamına gelir. 5G'nin altyapısı diğer kablosuz teknolojilerin altyapısından oldukça farklı olduğundan dolayı MIMO antenlerin baştan tasarlanması gerekir. Milimetre dalga anten tasarlamak oldukça zor bir iştir (Manan vd., 2019).

İlk etapta, birkaç kablosuz cihazın aynı anda çalışması için 5G uygulamalarında daha geniş bant genişliği gereksinimi gereklidir. Ayrıca, yüksek frekanslarda zayıflama arttığından ve buna bağlı olarak emilim etkileri de artacağından dolayı bu parametreler önemlidir. Bu nedenle, daha yüksek iletim kapasitesine ek olarak mm-dalga frekanslarındaki artan atmosferik zayıflamaları hesaba katmak için yüksek kazanç elde etmek esastır (Perić vd., 2016; Shayea vd., 2018; Nandi ve Maitra, 2018). Kompaktlık milimetre dalga frekanslarında önemli bir gereksinimdir. $\mathrm{Bu}$ nedenle antenin boyutunu küçük tutmak gerekir. Standart MIMO ağları, tek bir fiziksel pakette iki veya dört anten kullanma eğilimindedir (Ojaroudi Parchin vd., 2019). 5G teknolojisi günümüzdeki akıllı telefonlarda kullanılmaya başlandı. Fakat teorik olarak hesaplanan değerlere henüz ulaşılamamıştır. Bundan dolayı mobil telefonlarda kullanılan antenin kazanç değerine odaklanmak doğru bir yaklaşım olabilir. Bundan dolayı antenin kazanç değerleri üzerinde durulması tercih edilmiştir. Böylece yüksek frekanslarda ilerleyen sinyaller (örneğin $24 \mathrm{GHz}$ ) alıcıya ulaştığında daha güçlü ve daha temiz sinyaller elde edilebilir. $\mathrm{Bu}$ durum da hem konuşma kalitesini arttırırken hem de internet kalitesini arttırır.

Bu çalışmada, 5G cihazlar için, düşük profilli bir 4portlu MIMO anten dizisi önerilmiştir. MIMO anten sisteminin elemanları, bir dikdörtgen ve daha geniş iletim taşıma kapasitesine sahip dairesel bir yama antenidir. Burada seçilen dikdörtgensel ve dairesel yapının amacı, daha yüksek kazançlar elde etmek içindir. Önerilen anten, $1.1 \mathrm{GHz}$ geniş bant genişliğinde önemli miktarda kazanç sergilemektedir. MIMO anten tasarımının S parametreleri, verimliliği, radyasyon paterni, zarf korelasyon katsayis1 (Envelope Correlation Coefficient, ECC), toplam aktif yansıma katsayıs1 (Total Active Reflection Coefficient, TARC) açısından incelenmiştir. İncelenen frekans değerleri $5 \mathrm{G}$ teknolojisi için lisanslanmış aday frekans değerleridir. Bundan dolayı bu frekans değerlerine yoğunlaşılmıştır.

\section{Materyal ve metot}

Anten tasarımı, Şekil 1'de tek elemanlı anten, Şekil 2'de iki elemanlı anten ve Şekil 3'te $35 \times 50 \times 0.76 \mathrm{~mm}^{3}$ dielektrik malzeme üzerine yerleştirilen 4-portlu MIMO anten sistemi gösterilmiştir.

Tablo 1. Tasarlanan MIMO antenin milimetre $(\mathrm{mm})$ cinsinden parametre değerleri.

\begin{tabular}{cccccc}
\hline Parametre & Değer $\mathbf{( m m})$ & Parametre & Değer $\mathbf{( m m})$ & Parametre & Değer $(\mathbf{m m})$ \\
\hline cg & 1.2 & th & 1.45 & $\mathrm{dg}$ & 3.7 \\
cy & 3.1 & dkg & 1.7 & $\mathrm{dy}$ & 0.2 \\
hw & 0.4 & dgy & 2.5 & $\mathrm{dc}$ & 0.2 \\
hh & 3.6 & dus & 0.6 & ic & 0.5 \\
ig & 0.4 & & & & \\
\hline
\end{tabular}

Tablo 1'de tasarlanan MIMO antenin bir elemanl1 ve iki elemanlı boyutları $\mathrm{mm}$ cinsinden verilmiştir. 


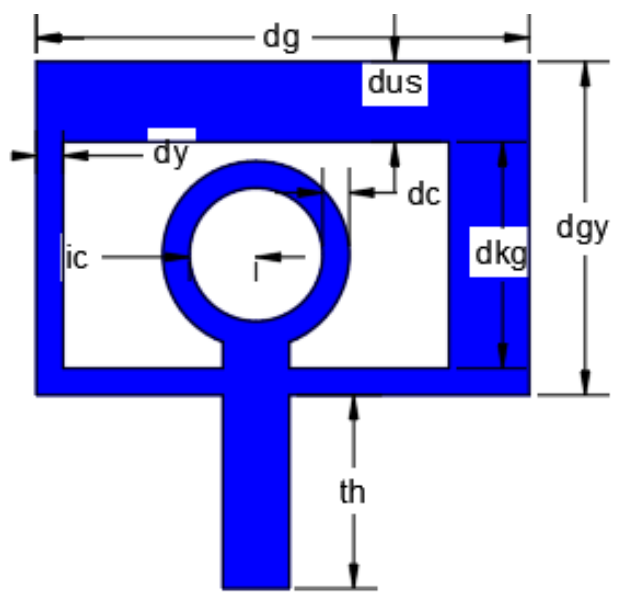

Şekil 1. Tek elemanlı anten yapısı ve ebatları

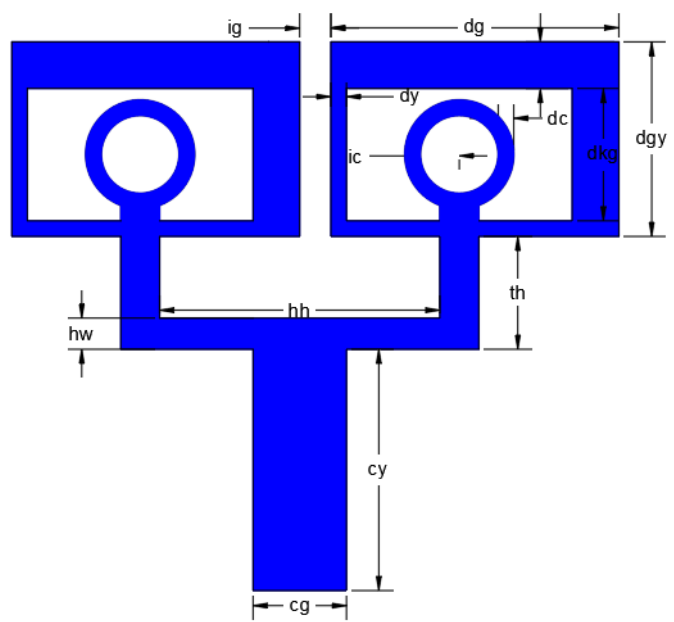

Şekil 2. İki elemanlı anten ve boyutları

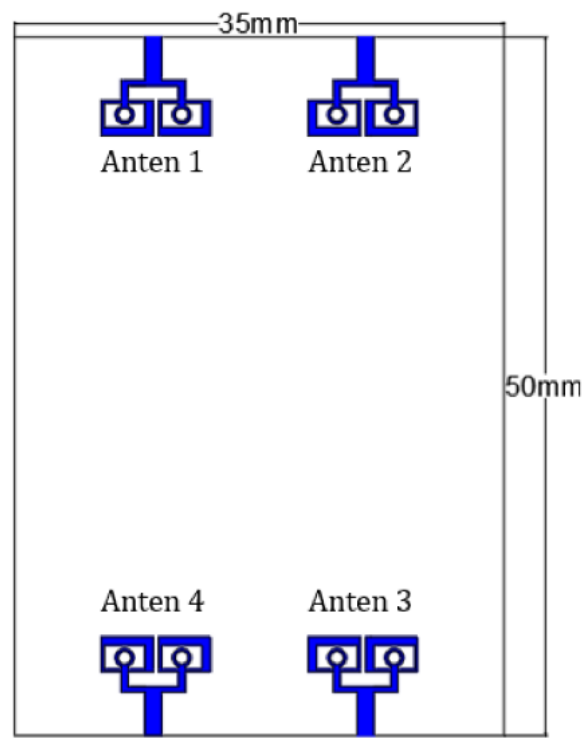

Şekil 3. $35 \times 50 \mathrm{~mm}^{2}$ dielektrik malzeme üzerine yerleştirilen 4-portlu MIMO anten sistemi

Geliştirilen antenin benzetimleri $24 \mathrm{GHz}, 27 \mathrm{GHz}$ ve $28 \mathrm{GHz}$ olmak üzere üç farklı frekans bandında gerçekleştirilmiştir. Antende, Rogers RT/duroid 5880 dielektrik malzemesi kullanılmıştır ve bağıl dielektrik katsayıs $\varepsilon_{r}=2.2$ olarak belirlenmiştir. Dielektrik tanjant kaybı 0.0009'dur ve kalınlığı $0.76 \mathrm{~mm}$ olarak belirlenmiştir. Ana port besleme empedans değeri $50 \Omega$ ve port giriş güçleri $1 \mathrm{~W}^{\prime}$ tır. Dielektrik malzemenin altındaki yüzey (ground panel) $35 \times 50 \times 0.3 \mathrm{~mm}^{3}$ ebatlarında bakır panel ile, tek elemanlı antenin yüzeyi $3.7 \times 4.15 \times 0.15 \mathrm{~mm}^{3}$ ebatlarında bakır panel ile ve iki elemanlı antenin yüzeyi $7.8 \times 7.05 \times 0.15 \mathrm{~mm}^{3}$ ebatlarında bakır panel ile kaplanmıştır. Ayrıca MIMO antenin, ECC ve TARC değerleri de çıkarılmıştır.

\subsection{Zarf korelasyon katsaylsı (Envelope correlation coefficient, ECC)}

ECC, MIMO sistemlerinin temel başarım parametrelerinden biridir (Sharawi, 2013) ve Denklem (1) (Ojaroudi Parchin vd., 2019) kullanılarak hesaplanır.

$$
E C C=\frac{\left|S_{m m}^{*} S_{n m}+S_{m n}^{*} S_{n n}\right|^{2}}{\left(1-\left|S_{m m}\right|^{2}-\left|S_{m n}\right|^{2}\right)\left(1-\left|S_{n m}\right|^{2}-\left|S_{n n}\right|^{2}\right)}
$$

ECC değerinin, genel anten modülünde pratik standardının 0.5 'in altında olması gerekir (Khalid vd., 2020).

\subsection{Toplam aktif yansima katsaylsı (Total active reflection coefficient, TARC)}

Toplam aktif yansıma katsayısı, özellikle MIMO anten sistemlerinde, çok portlu antenlerin geri dönüş kaybı olarak tanımlanabilir (Marzudi vd., 2014) ve Denklem (2) kullanılarak hesaplanır.

$T A R C=-\sqrt{\frac{\left(S_{m m}+S_{m n}\right)^{2}+\left(S_{n m}+S_{n n}\right)^{2}}{2}}$

Buradaki $\mathrm{S}$ ifadesi $\mathrm{S}$ parametre değerlerinden gelmektedir. $\mathrm{m}=1,2,3, \ldots$ ve $\mathrm{n}=1,2,3, \ldots$ şeklinde devam etmektedir.

\section{Bulgular ve tartışma}

ANSYS HFSS $®$ bilgisayar simülatörü sonuçlarına göre geliştirilen MIMO antenin kazancı en yüksek $27 \mathrm{GHz}$ frekans bandında olup, bu değer $13.05 \mathrm{~dB}$ olarak elde edilmiştir. Tasarımı gerçekleştirilen MIMO anten, 5G mobil cep telefonlarında, 5G WiFi cihazlarında ve akıllı kol saatlerinde (2 elemanlı versiyonu) kullanılabilir. 


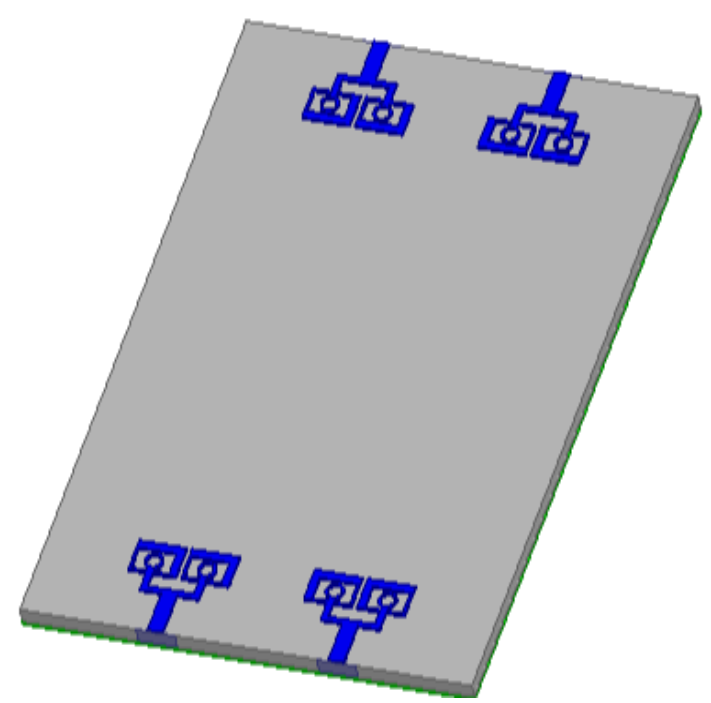

Şekil 4. Geliştirilen MIMO antenin üç boyutlu (3B) hali

Şekil 4'te 4-portlu MIMO antenin üç boyutlu tasarımı verilmiştir.

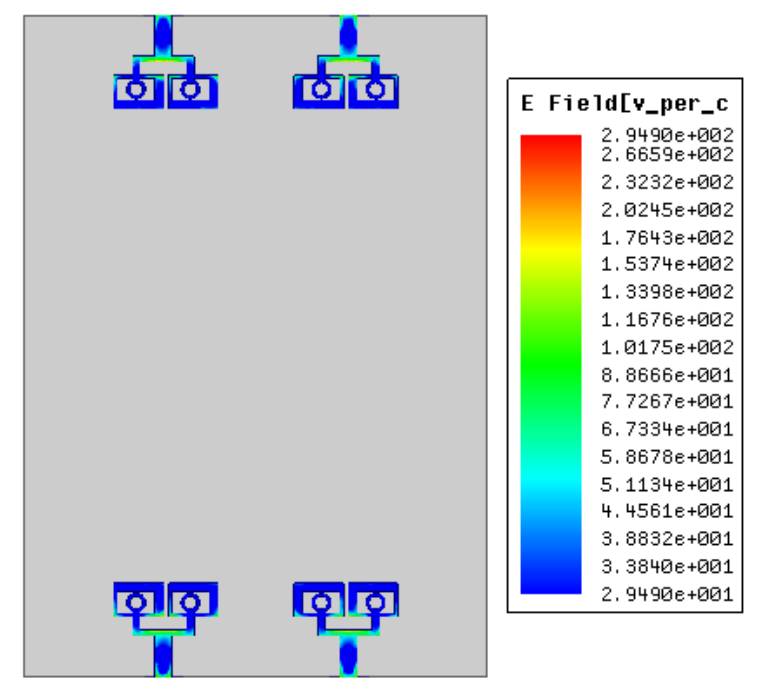

Şekil 5. 24GHz'de elektrik alan dağılımı
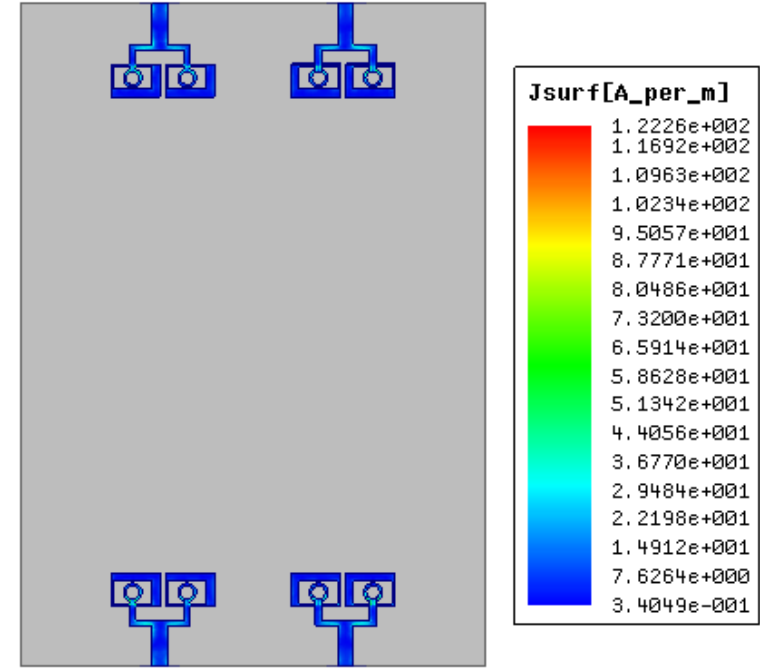

Şekil 6. $24 \mathrm{GHz}$ 'de antenin yüzey akım dağılımı

Şekil 5'te MIMO antenin elektrik alan dağılımı ve Şekil 6'da MIMO antenin yüzey akım dağılımları verilmiştir. Maksimum elektrik alan yoğunluğu $294 \mathrm{~V} / \mathrm{cm}$ iken maksimum yüzey akım yoğunluğu ise $122 \mathrm{~A} / \mathrm{m}$ olarak simüle edilmiştir. Şekil 7, Şekil 8 ve Şekil 9'da $24 \mathrm{GHz}, 27 \mathrm{GHz}$ ve $28 \mathrm{GHz}$ frekans bantlarında normalize edilmiş $\varphi$ açısı $0^{\circ}$ ve $90^{\circ}$ iken 4-portlu MIMO antenin oluşturduğu radyasyon paternleri gösterilmiştir. $24 \mathrm{GHz}$ 'de radyasyonun oluşturduğu maksimum büyüklük $\varphi$ açısı $0^{\circ}$ iken $10^{\circ}$ lik, $\varphi$ açısı $90^{\circ}$ iken $-60^{\circ}$ lik yönelme açıları ile maksimum büyüklükleri sirasiyla $-0.0247 \mathrm{~dB}$ ve $-0.0287 \mathrm{~dB}, 27 \mathrm{GHz}$ 'de radyasyonun oluşturduğu maksimum büyüklük $\varphi$ açısı $0^{\circ}$ iken $-10^{\circ}$ lik, $\varphi$ açısı $90^{\circ}$ iken $-50^{\circ}$ 'lik yönelme açıları ile maksimum büyüklükleri sirasiyla $-0.0897 \mathrm{~dB}$ ve $-0.1 \mathrm{~dB}$ ve $28 \mathrm{GHz}$ 'de radyasyonun oluşturduğu maksimum büyüklük $\varphi$ açısı $0^{\circ}$ iken $-10^{\circ}$ 'lik, $\varphi$ aç1sı $90^{\circ}$ iken $50^{\circ}$ 'lik yönelme açıları ile maksimum büyüklükleri sirasiyla $-0.181 \mathrm{~dB}$ ve $-0.0924 \mathrm{~dB}$ olarak gözlemlenmiştir.
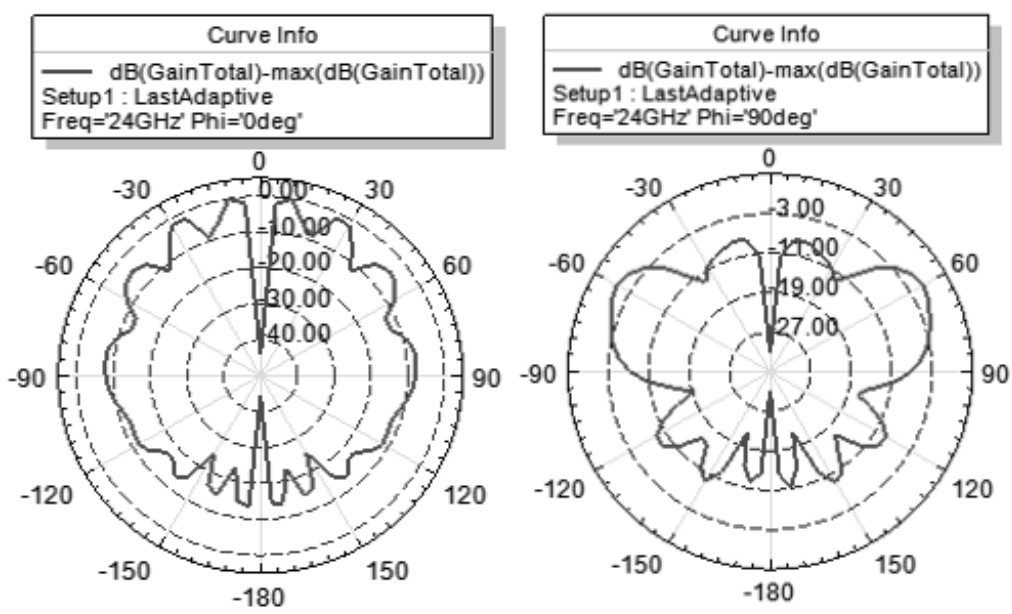

Şekil 7. Normalize edilen E-düzlemi $\left(\varphi=0^{\circ}\right)$ ve H-düzlemindeki $\left(\varphi=90^{\circ}\right) 24 \mathrm{GHz}$ için simüle edilen $2 \mathrm{~B}$ radyasyon paterni 

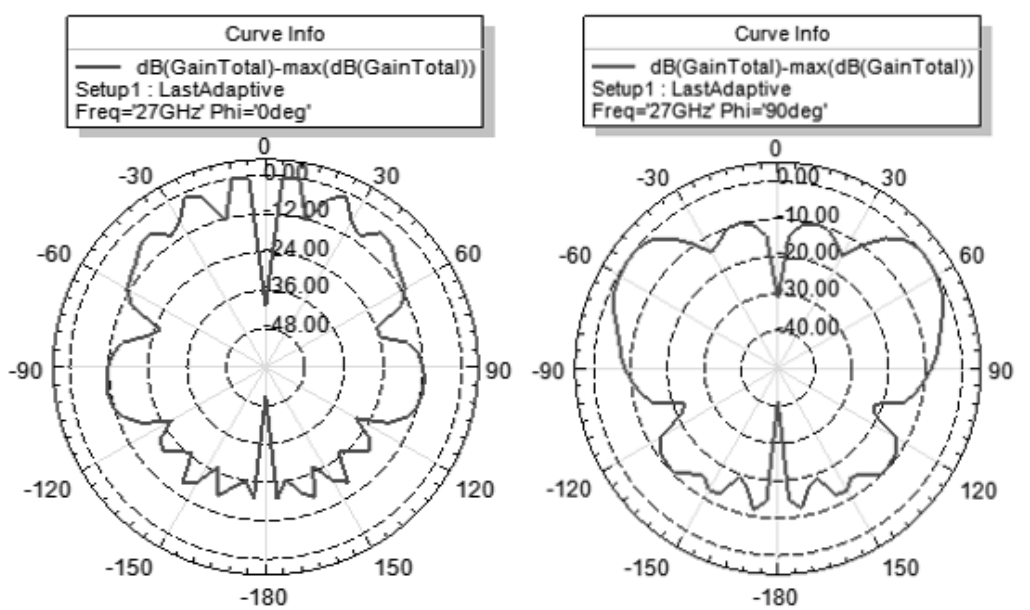

Şekil 8. Normalize edilen E-düzlemi $\left(\varphi=0^{\circ}\right)$ ve H-düzlemindeki $\left(\varphi=90^{\circ}\right) 27 \mathrm{GHz}$ için simüle edilen $2 \mathrm{~B}$ radyasyon paterni
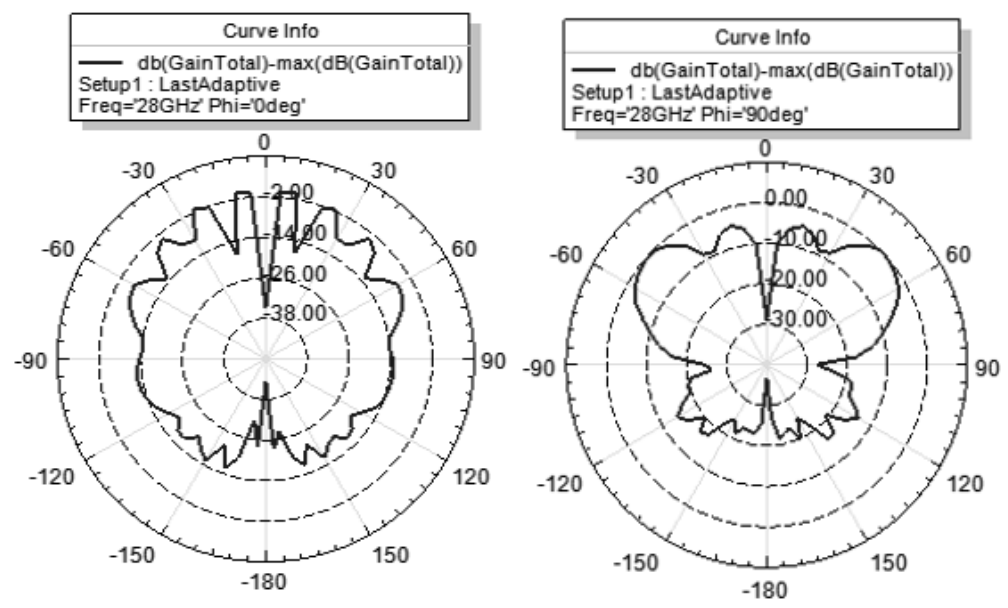

Şekil 9. Normalize edilen E-düzlemi $\left(\varphi=0^{\circ}\right)$ ve H-düzlemindeki $\left(\varphi=90^{\circ}\right) 28 \mathrm{GHz}$ için simüle edilen $2 \mathrm{~B}$ radyasyon paterni

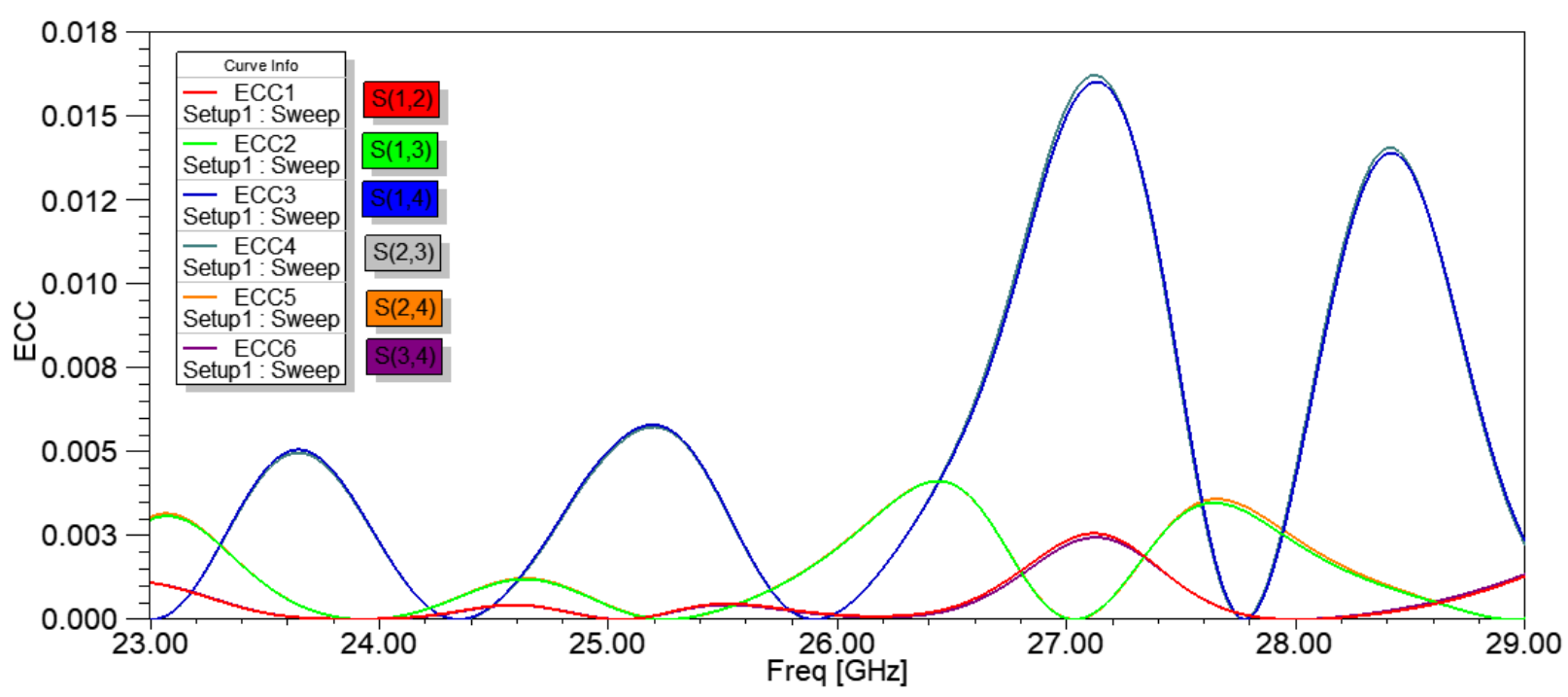

Şekil 10. Önerilen MIMO antenin $S_{12}, S_{13}, S_{14}, S_{23}, S_{24}$ ve $S_{34}$ zarf korelasyon katsayıları

Şekil 10'da önerilen MIMO anten dizisinin ECC grafiği frekans spektrumda verilmiştir. Bütün frekans bandında $\mathrm{S}$ parametrelerinin ECC katsayıları 0.017'den daha küçüktür. Bu durumda 
ECC katsayısı 0.5 değerinden çok çok düşük olduğundan, 4 anten elemanının da birbirlerinden ilgisiz olduğunu gösterir.

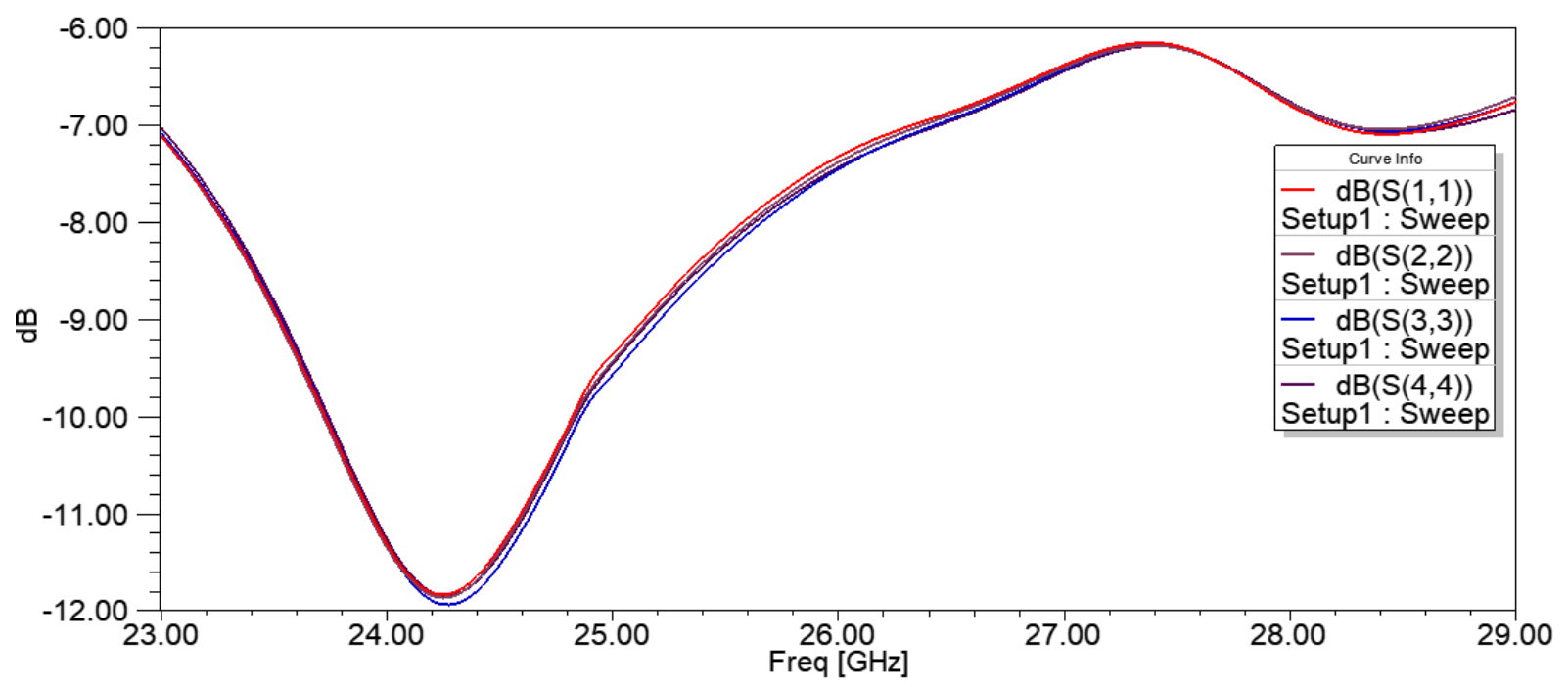

Şekil 11. Antenin $S_{11}, S_{22}, S_{33}$ ve $S_{44}$ parametre sonuçları

Şekil 11'de önerilen MIMO antenin $\mathrm{S}_{11}, \mathrm{~S}_{22}, \mathrm{~S}_{33}$ ve $\mathrm{S}_{44}$ parametre sonuçları frekans spektrumunda verilmiştir. Görüldüğü üzere 4 anten için de parametre değerleri yakındır. Değerler negatif ($6 \mathrm{~dB}$ ile $-12 \mathrm{~dB}$ arasında) olduğundan dolayı aynı zamanda iyi bir değere sahiptir.

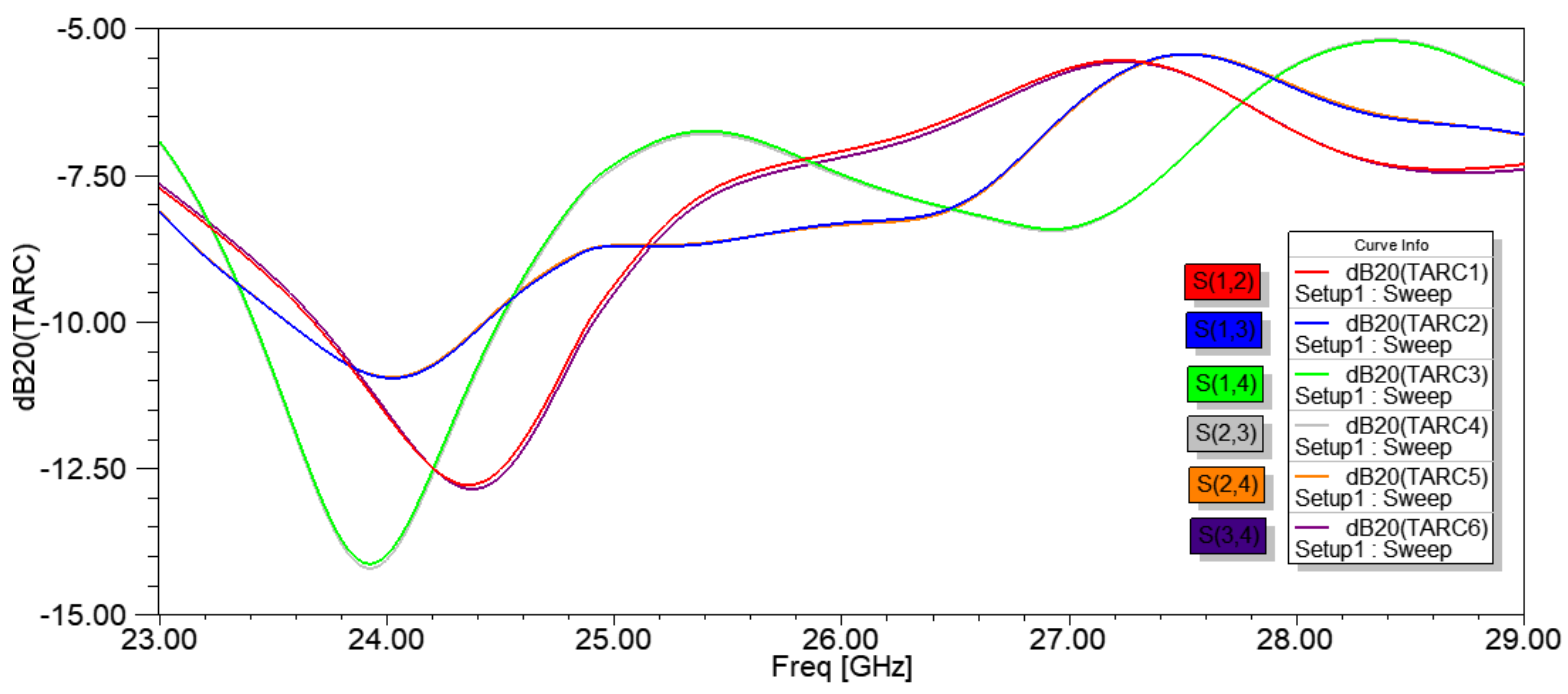

Şekil 12. Önerilen MIMO antenin $S_{12}, S_{13}, S_{14}, S_{23}, S_{24}$ ve $S_{34}$ parametrelerinin aktif yansıma katsay1ları

Şekil 12'de önerilen MIMO anten dizisinin TARC grafiği frekans spektrumda verilmiştir. TARC değerinin minimum değeri $\mathrm{S}(2,3)$ parametresinde olup 23.92GHz'dedir. Buradan, TARC ve ECC değerlerinin daha düşük kapasite kaybına ve daha iyi başarıma sahip oldukları söylenebilir.

Tablo 2. 5G aday frekanslarındaki tekli antenlerin kazançları, toplam MIMO anten kazancı, maksimum verimlilik ve maksimum yönelme değerleri

\begin{tabular}{cccccccc}
\hline $\begin{array}{c}\text { Çalışma } \\
\text { Frekansı } \\
(\mathbf{G H z})\end{array}$ & $\begin{array}{c}\text { Ante } \\
\mathbf{n ~ 1}\end{array}$ & $\begin{array}{c}\text { Kazanç(dB) } \\
\text { Anten 2 }\end{array}$ & Anten 3 & Anten 4 & $\begin{array}{c}\text { Toplam } \\
\text { Anten } \\
\text { Kazancı (dB) }\end{array}$ & $\begin{array}{c}\text { Maksimum } \\
\text { Yönelme } \\
\text { (dB) }\end{array}$ & $\begin{array}{c}\text { Maksimum } \\
\text { Verimilik } \\
(\%)\end{array}$ \\
\hline 24 & 8.67 & 8.66 & 8.65 & 8.78 & 12.70 & 13.44 & 94.50 \\
27 & 8.85 & 8.87 & 8.94 & 8.91 & 13.05 & 14.37 & 91.81 \\
28 & 8.33 & 8.21 & 8.27 & 8.34 & 10.48 & 12.81 & 81.81 \\
\hline
\end{tabular}


Tablo 2'de 5G için üç farklı aday frekans bantları ve bu bantlarda her bir antenin oluşturduğu kazanç, 4-portlu MIMO antenin oluşturduğu maksimum kazanç, maksimum verimlilik ve maksimum yönelme değerleri verilmiştir.

Tablo 3. Literatürdeki çalışmalar ile mevcut çalışmanın karşılaştırılması

\begin{tabular}{|c|c|c|c|c|c|c|c|}
\hline Kaynak & $\begin{array}{l}\text { Frekans } \\
(\mathbf{G H z})\end{array}$ & $\begin{array}{l}\text { Port } \\
\text { Sayisı }\end{array}$ & Boyut $\left(\mathrm{mm}^{\mathbf{3}}\right)$ & $\begin{array}{c}\text { Bant } \\
\text { Genişliği } \\
\text { (GHz) }\end{array}$ & $\begin{array}{l}\text { Kazanç } \\
\text { (dBi) }\end{array}$ & $\begin{array}{l}\text { Verimlilik } \\
(\%)\end{array}$ & ECC \\
\hline $\begin{array}{c}\text { [Ojaroudi Parchin } \\
\text { vd., 2019] }\end{array}$ & 3.6 & 8 & $150 \times 75 \times 1.6$ & 1.2 & 2.5 & $60-80$ & $<0.01$ \\
\hline [Khalid vd., 2020] & 28 & 4 & $30 \times 35 \times 0.76$ & 4.1 & $8.3 \mathrm{~dB}$ & 82 & $<0.01$ \\
\hline [Iqbal vd., 2019] & 24 & 2 & $15 \times 19 \times 0.254$ & 0.8 & 6 & 80.5 & 0.24 \\
\hline [Park vd., 2016] & 28 & 4 & Belirtilmemiş & 1.5 & 7.41 & Belirtilmemiş & Belirtilmemiş \\
\hline $\begin{array}{c}\text { [Sun ve Leung, } \\
\text { 2016] }\end{array}$ & 24 & 2 & $40 \times 25 \times 0.254$ & 0.77 & 7.37 & Belirtilmemiş & Belirtilmemiş \\
\hline [Sharawi vd., 2017] & 30 & 2 & $48 \times 21 \times 0.13$ & 1 & $>7$ & 80 & $<0.4$ \\
\hline [Zhang vd., 2019] & 28 & 2 & $20 \times 20 \times 0.254$ & 0.85 & $8 \mathrm{~dB}$ & Belirtilmemiş & 0.013 \\
\hline [Shoaib vd., 2018] & 24 & 8 & $31.2 \times 31.2 \times 1.57$ & 5.68 & 7.86 & 78.9 & 0.04 \\
\hline [Bu Çalışma] & 24 & 4 & $35 \times 50 \times 0.76$ & 1.1 & 12.70 & 94.50 & $<0.017$ \\
\hline
\end{tabular}

Tablo 3'te, üzerinde çalışılan bütün frekans bantları incelendiğinde, bu çalışmada geliştirilen 4portlu MIMO antenin diğer çalışmalardaki MIMO antenlere göre kazancının, verimliliğinin ve bazı
ECC değerine göre daha iyi olduğu söylenebilir. Ancak tasarım gerçekleşmediğinden dolayı antendeki kazanç değerleri benzetim değerlerine yakın çıkmayabilir.

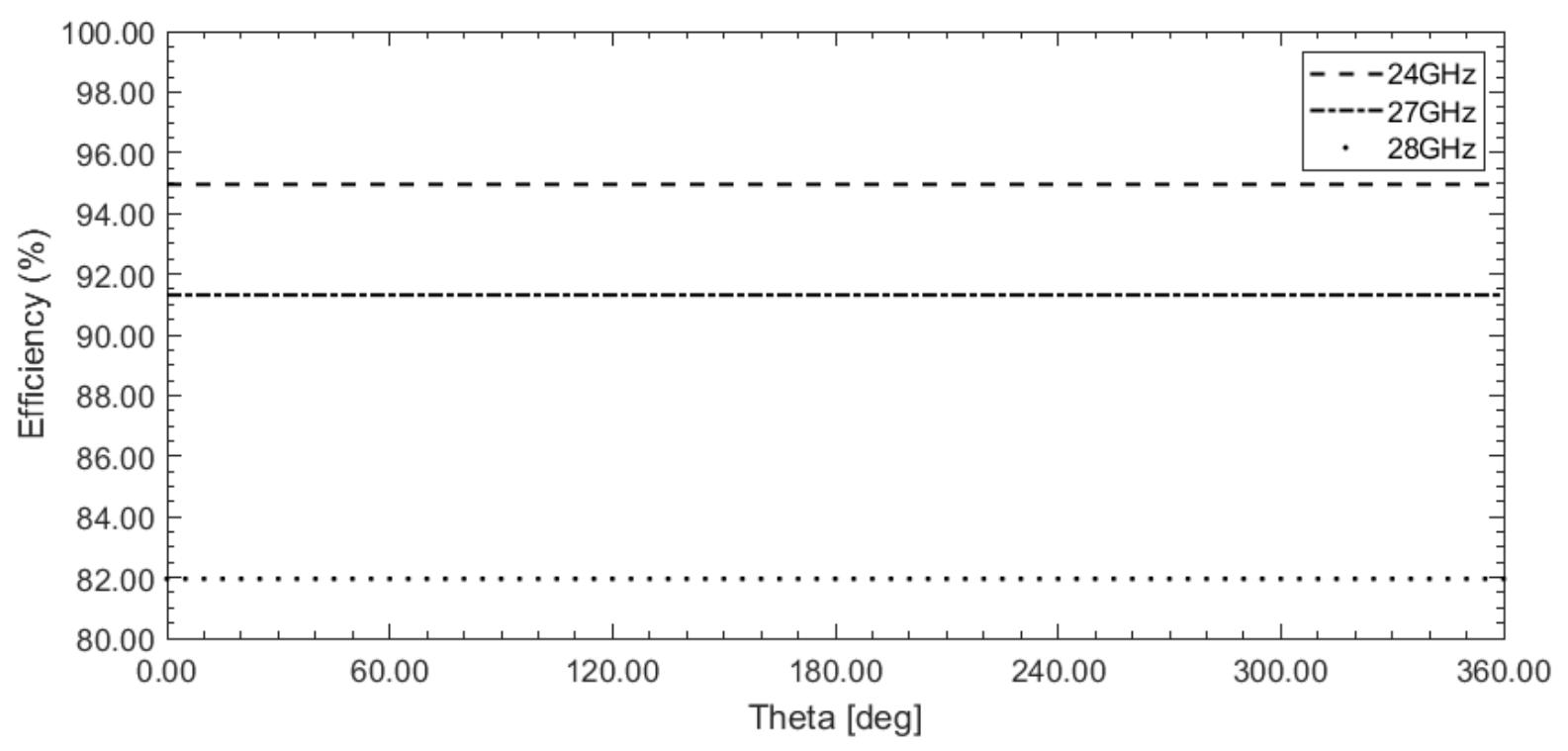

Şekil 13. 4-portlu MIMO antenin toplam maksimum verimliliği

Şekil 13’te 4-portlu MIMO antenin yüzdesel olarak toplam verimliliği gösterilmiştir. Verimlilik değeri bütün frekans spektrumunda incelendiğinde, verimlilik değeri en yüksek $24 \mathrm{GHz}$ frekansında olup, verimliliği \%94.50'dir. Bu değer, geliştirilen MIMO anten iyi bir verimlilik değerdir. $27 \mathrm{GHz}$ 'de verimlilik değeri $\% 91.81$ ve $28 \mathrm{GHz}$ 'de verimlilik değeri \%81.81 olarak bulunmuştur.

Şekil 14'te gösterildiği gibi $\mathrm{S}_{11} \leq-10 \mathrm{~dB}$ altında kalan frekans değerlerine göre yani $23.7 \mathrm{GHz}-$
$24.8 \mathrm{GHz}$ arasında olup, buradan bant genişliği $1.1 \mathrm{GHz}$ olarak bulunur.

Şekil 15, Şekil 16 ve Şekil 17'de geliştirilen MIMO antenin üç frekans bandında da $\mathrm{dB}$ olarak toplam kazanç değerlerinin 3B 1şıma dağılımları verilmiştir. Geliştirilen MIMO anten, en çok kazanca $27 \mathrm{GHz}$ 'de, daha sonra $24 \mathrm{GHz}$ 'de ve en az $28 \mathrm{GHz}$ 'de sahiptir. 


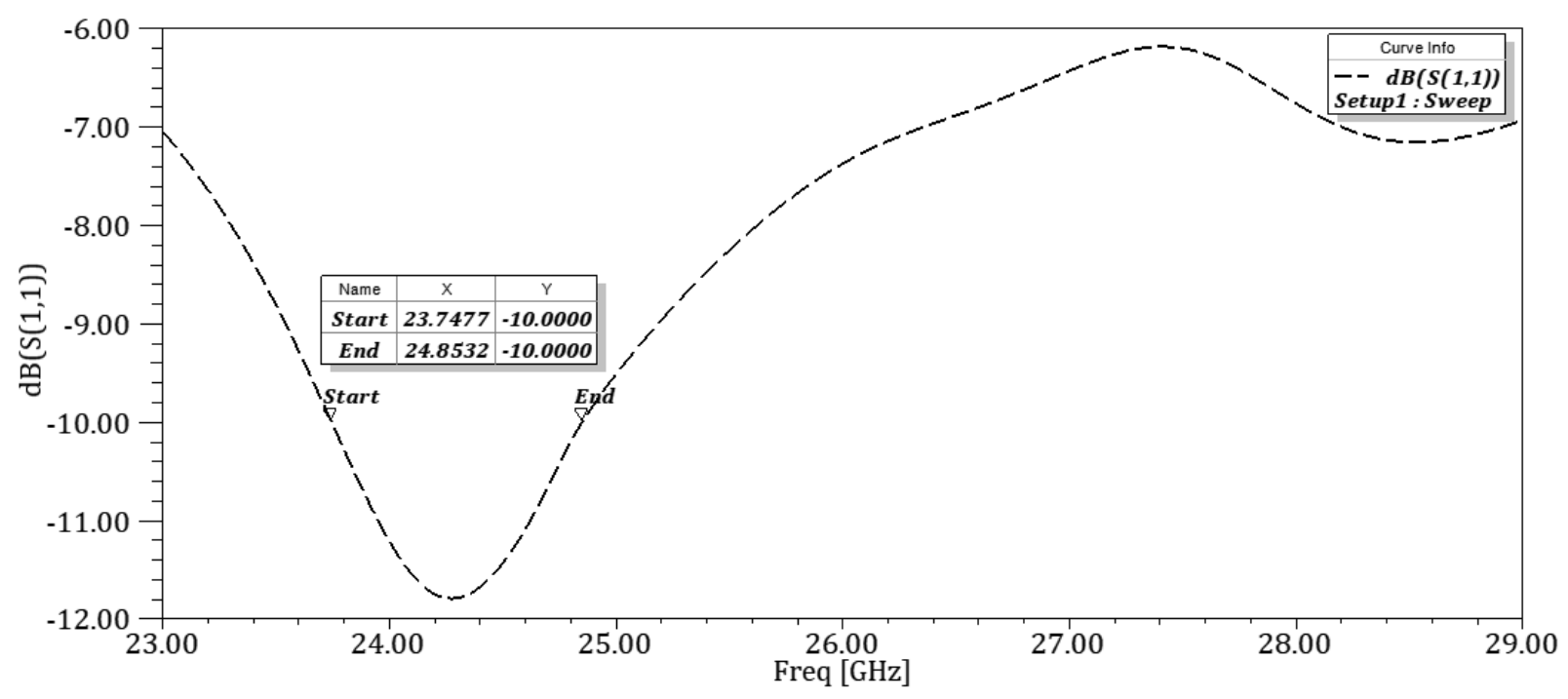

Şekil 14. MIMO antenin bant genişliği

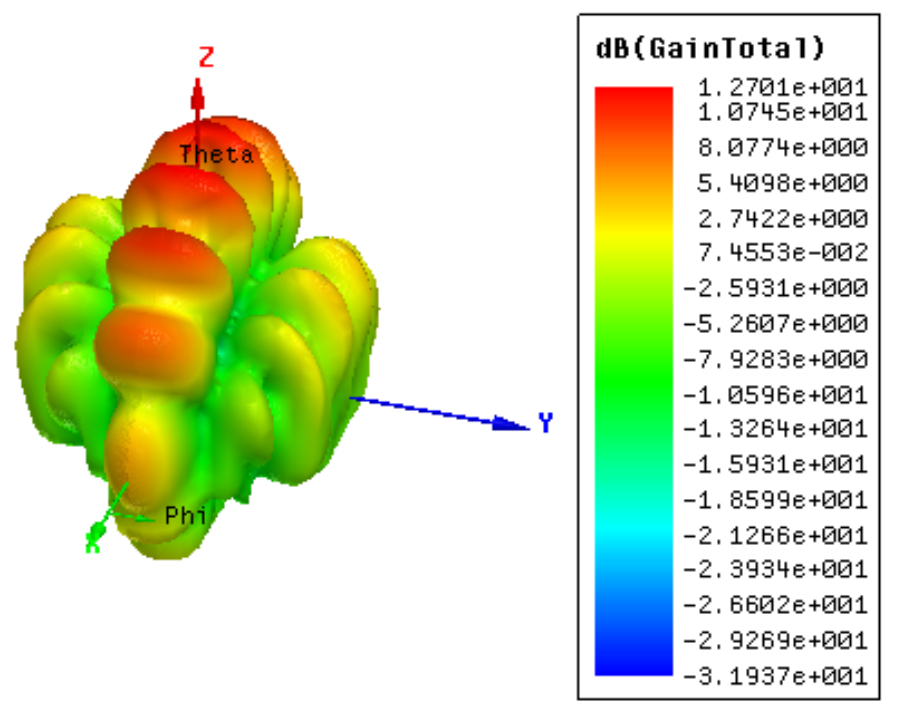

Şekil 15. $24 \mathrm{GHz}$ için toplam anten kazancı 3B 1şıması

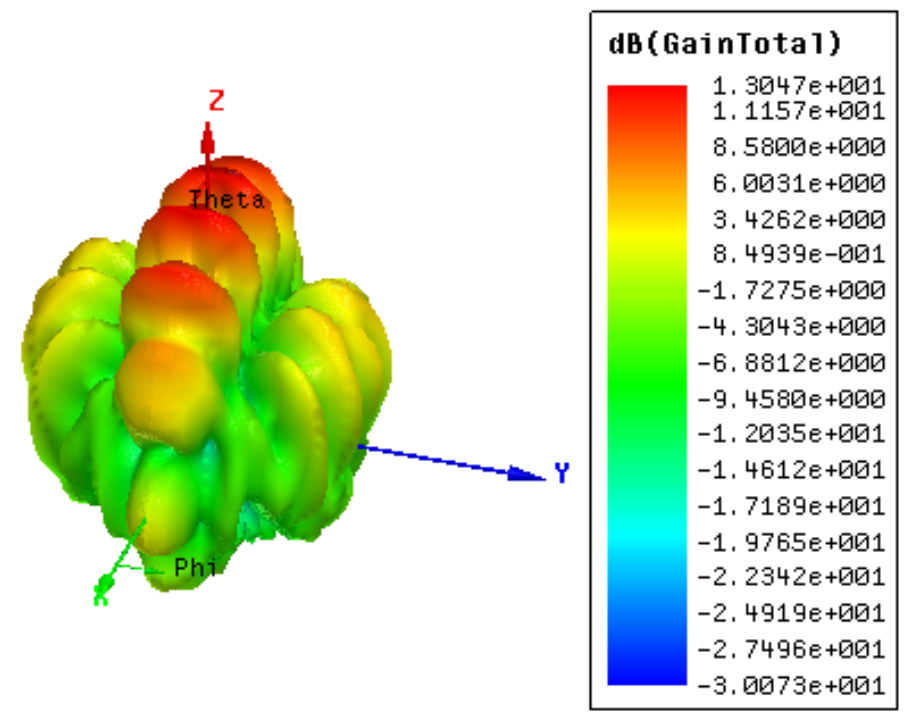

Şekil 16. 27GHz için toplam anten kazancı 3B 1şıması 


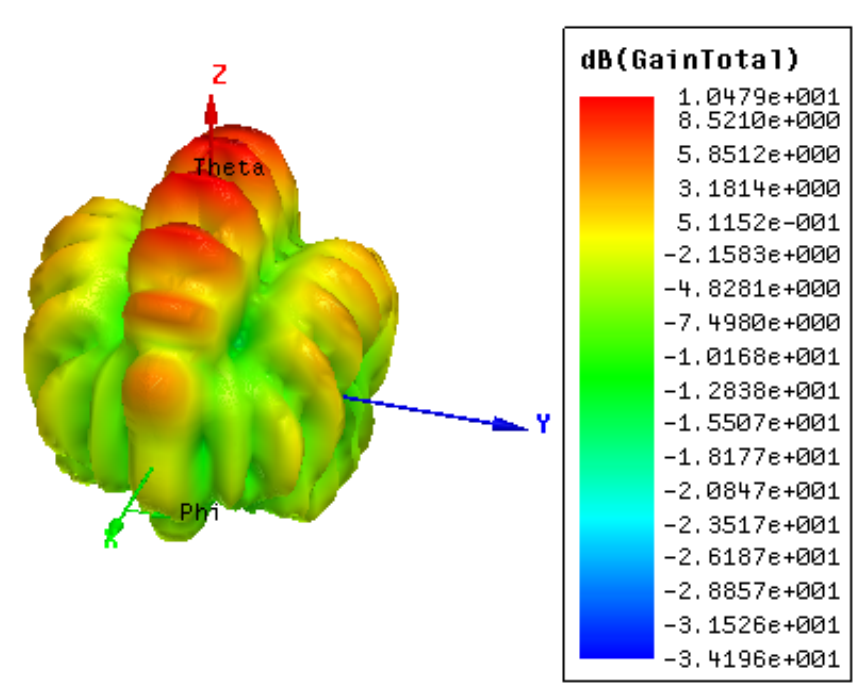

Şekil 17. 28GHz için toplam anten kazancı 3B 1şıması

\section{Sonuçlar}

$\mathrm{Bu}$ çalışmada 5G aday frekanslarında kullanılmak üzere 4-portlu MIMO anten tasarımı yapılmış ve incelenmiştir. Elde edilen sonuçlara göre $24 \mathrm{GHz}$ 'de kazanç değeri $12.7 \mathrm{~dB}$ olarak bulunmuştur. Böylece anten, bu frekans bandında veya bu frekansa yakın değerlerde çalışacağ 1 düşünülmektedir. Antenin bant genişliği $\mathrm{S}_{11}$ parametresinden bulunmuştur ve planlanan $5 \mathrm{G}$ aday bant genişliği sınırları içerisinde çıktığı görülmüştür. Verimlilik bakımından da incelenen bu anten, 24GHz'de \%94.50'lik bir oranla iyi bir anten modeli olduğu söylenebilir.

\section{Kaynaklar}

Iqbal, A., Basir, A., Smida, A., Mallat, N. K., Elfergani, I., Rodriguez, J. and Kim, S. (2019). Electromagnetic bandgap backed millimeterwave MIMO antenna for wearable applications. IEEE Access, 7, 111135-111144. https://doi.org/10.1109/ACCESS.2019.2933913

Khalid, M., Iffat Naqvi, S., Hussain, N., Rahman, M., Mirjavadi, S.S., Khan, M. J. and Amin, Y. (2020). 4-Port MIMO antenna with defected ground structure for $5 \mathrm{G}$ millimeter wave applications. Electronics, 9(1), 71. https://doi.org/10.3390/electronics9010071

Manan, A., Naqvi, S. I., Azam, M. A., Amin, Y., Loo, J. and Tenhunen, H. (2019). MIMO Antenna array for mm-wave 5G smart devices. In2019 22nd International Multitopic Conference (INMIC) (pp. 1-5). IEEE. https://doi.org/10.1109/INMIC48123.2019.9022 757

Marzudi, W. N. N. W., Abidin, Z. Z., Muji, S. Z. M., Yue, M. and Abd-Alhameed, R. A. (2014).
Minimization of mutual coupling using neutralization line technique for $2.4 \mathrm{GHz}$ wireless applications. https://doi.org/10.17781/P001280

Nandi, D., and Maitra, A. (2018). Study of rain attenuation effects for $5 \mathrm{G} \mathrm{Mm}$-wave cellular communication in tropical location. IET Microwaves, Antennas \& Propagation, 12(9), 1504-1507. https://doi.org/10.1049/ietmap.2017.1029

Ojaroudi Parchin, N., Jahanbakhsh Basherlou, H., Alibakhshikenari, M., Ojaroudi Parchin, Y., AlYasir, Y. I., Abd-Alhameed, R. A. and Limiti, E. (2019). Mobile-phone antenna array with diamond-ring slot elements for $5 \mathrm{G}$ massive MIMO systems. Electronics, 8(5), 521. https://doi.org/10.3390/electronics8050521

Park, J. S., Ko, J. B., Kwon, H. K., Kang, B. S. Park, B. and Kim, D. (2016). A tilted combined beam antenna for $5 \mathrm{G}$ communications using a $28-\mathrm{GHz}$ band. IEEE Antennas and Wireless Propagation Letters, $15, \quad 1685-1688$. https://doi.org/10.1109/LAWP.2016.2523514

Perić, M. V., Perić, D. B., Todorović, B. M. and Popović, M. V. (2016). Dynamic rain attenuation model for millimeter wave network analysis. IEEE Transactions on Wireless Communications, 16(1), 441-450. https://doi.org/10.1109/TWC.2016.2624729

Rajo-Iglesias, E. and Sharawi, M. S. (2016). MIMO Antennas. in wideband, multiband, and smart reconfigurable antennas for modern wireless communications (pp. 145-175). IGI Global.

Sharawi, M. S. (2013). Printed multi-band MIMO antenna systems and their performance metrics [wireless corner]. IEEE Antennas and 
Propagation Magazine, 55(5), 218-232. https://doi.org/10.1109/MAP.2013.6735522

Sharawi, M. S. (2017). Advancements in MIMO antenna systems. Developments in Antenna Analysis and Synthesis.

Sharawi, Mohammad S., Symon K. Podilchak, Mohamed T. Hussain, and Yahia MM Antar (2017). Dielectric resonator based MIMO antenna system enabling millimetre-wave mobile devices. IET Microwaves, Antennas \& Propagation 11, (2), 287-293. https://doi.org/10.1049/iet-map.2016.0457

Shayea, I., Rahman, T. A., Azmi, M. H. and Islam, M. R. (2018). Real measurement study for rain rate and rain attenuation conducted over $26 \mathrm{GHz}$ microwave $5 \mathrm{G}$ link system in Malaysia.IEEE
Access,

6 ,

19044-19064 https://doi.org/10.1109/ACCESS.2018.2810855

Shoaib, N., Shoaib, S., Khattak, R. Y., Shoaib, I., Chen, X. and Perwaiz, A. (2018). MIMO antennas for smart 5G devices. IEEE Access, 6, 77014-77021. https://doi.org/10.1109/ACCESS.2018.2876763

Sun, Y. X. and Leung, K. W. (2016). Substrateintegrated two-port dual-frequency antenna. IEEE Transactions on Antennas and Propagation, 64(8), 3692-3697. https://doi.org/10.1109/TAP.2016.2565740

Zhang, Y., Deng, J. Y., Li, M. J., Sun, D. and Guo, L. X. (2019). A MIMO dielectric resonator antenna with improved isolation for $5 \mathrm{G} \mathrm{mm-wave}$ applications. IEEE Antennas and Wireless Propagation Letters, 18(4), 747-751. doi: 10.1109/LAWP.2019.2901961 